\title{
The Threshold of a Stochastic SIRS Model with Vertical Transmission and Saturated Incidence
}

\author{
Chunjuan Zhu, Guangzhao Zeng, and Yufeng Sun \\ School of Mathematics and Statistics, Shaoguan University, Shaoguan 512005, China \\ Correspondence should be addressed to Yufeng Sun; suny2001@sina.com
}

Received 27 January 2016; Accepted 15 November 2016; Published 28 February 2017

Academic Editor: Manuel De la Sen

Copyright (C) 2017 Chunjuan Zhu et al. This is an open access article distributed under the Creative Commons Attribution License, which permits unrestricted use, distribution, and reproduction in any medium, provided the original work is properly cited.

The threshold of a stochastic SIRS model with vertical transmission and saturated incidence is investigated. If the noise is small, it is shown that the threshold of the stochastic system determines the extinction and persistence of the epidemic. In addition, we find that if the noise is large, the epidemic still prevails. Finally, numerical simulations are given to illustrate the results.

\section{Introduction}

Mathematical models can describe the progress of a disease and predict the trend of the disease and they can provide the theoretical basis for people to undertake prevention strategies. At present, researches have constructed a series of mathematical models [1-10], including SIRS model $[5,9,10]$. They divided people into susceptible, $S$, infective, $I$, and removed, $R$, categories and one of the most famous SIRS epidemic models is the following:

$$
\begin{aligned}
S^{\prime}(t)= & \Lambda+b(S(t)+I(t)+R(t))-\beta S(t) I(t) \\
& -\mu S(t)+\varepsilon R(t), \\
I^{\prime}(t)= & \beta S(t) I(t)-(\mu+a+\gamma) I(t), \\
R^{\prime}(t)= & \gamma I(t)-(\mu+\varepsilon) R(t) .
\end{aligned}
$$

Here, $\Lambda$ is the birth rate, $\beta$ is the transmission rate, $b$ and $\mu$ are the birth rate and natural death rate, respectively, of $S, I$, and $R$ individuals, $a$ is the death rate of the disease, and $\gamma$ is the recovery rate of the infective individuals. Assume $\mu>b$.

Medical research has shown that the herpes virus will be in the form of mother-to-child transmission (vertical transmission) to the baby. In addition, since susceptible individuals in contact with every infective individual are limited, we see that when the number of the susceptible individuals is large, the bilinear incidence $\beta S I$ is unreasonable to consider. In this case, saturated incidence is more suitable than bilinear incidence [11].

In this paper, the transmission rate is chosen as the saturated incidence rate $\beta S I /(1+\alpha I)$, and the SIRS model is described as the following:

$$
\begin{aligned}
& S^{\prime}(t)=\Lambda+b(S(t)+R(t))+(1-q) b I-\frac{\beta S(t) I(t)}{1+\alpha I(t)} \\
&-\mu S(t)+\varepsilon R(t), \\
& I^{\prime}(t)= \frac{\beta S(t) I(t)}{1+\alpha I(t)}+q b I(t)-(\gamma+a+\mu) I(t), \\
& R^{\prime}(t)= \gamma I(t)-(\mu+\varepsilon) R(t) .
\end{aligned}
$$

In system (2), $q$ is vertical transmission rate, $R_{0}=\beta \Lambda /(\mu-$ $b)(\mu+\gamma+a-q b)$ is the threshold which determines whether the disease will die out or persist, and there always is a disease-free equilibrium $P^{0}=(\Lambda /(\mu-b), 0,0)$; see $[5,9,10]$ and the references therein. When $R_{0}<1$, the disease-free equilibrium $P^{0}$ is globally asymptotically stable; when $R_{0}>1$, $P^{0}$ is unstable and there is an endemic equilibrium $P^{*}=$ $\left(S^{*}, I^{*}, R^{*}\right)$ where $I^{*}=((\mu+\varepsilon)(\mu-b)(\mu+\gamma+a-q b) /(\beta(\mu-$ b) $\gamma+[\beta(\mu-b)+\beta+\alpha(\mu-b)(\mu+\gamma+a-q b)](\mu+\varepsilon)))\left(R_{0}-1\right)$, $R^{*}=(\gamma /(\mu+\varepsilon)) I^{*}, S^{*}=(\mu+\gamma+a-q b)\left(1+\alpha I^{*}\right) / \beta$, which is globally stable under a sufficient condition. 
However, all parameters in system (2) are affected by environmental noise, so it is of benefit to use a stochastic model. Stochastic models are more realistic compared to deterministic models. Many stochastic models for epidemic populations have been studied [12-19]. Tornatore et al. [18] studied an stochastic SIR model. They showed that under the condition $0<\beta<\min \left\{\gamma+\mu-\sigma^{2} / 2,2 \mu\right\}$, the disease-free equilibrium is locally stable, but the authors do not discuss under which condition the disease will prevail. Concerning the transmission coefficient $\beta$, Gray et al. [20] considered the stochastic SIS (susceptible-infective-susceptible) epidemic model with fluctuation. They proved threshold $R_{0}$ which determines the extinction and persistence of $I(t)$ according to the fluctuation. Here, $R_{0}$ is the threshold of the deterministic model; however, it is more difficult to get the threshold of the stochastic model.

We consider certain stochastic environmental factors and assume that fluctuations in the environment will manifest themselves mainly as fluctuations in the parameter $\beta$, as in [20],

$$
\beta \longrightarrow \beta+\dot{B}(t)
$$

where $B(t)$ is standard Brownian motion with $B(0)=0$ and $\sigma$ is the intensity. The stochastic version corresponding to the deterministic model (2) is the following:

$$
\begin{aligned}
& d S(t)=[\Lambda+b(S(t)+R(t))+(1-q) b I(t) \\
& \left.-\frac{\beta S(t) I(t)}{1+\alpha I(t)}-\mu S(t)+\varepsilon R(t)\right] d t-\sigma \\
& . \frac{S(t) I(t)}{1+\alpha I(t)} d B(t), \\
& d I(t)=\left[\frac{\beta S(t) I(t)}{1+\alpha I(t)}+q b I(t)-(\gamma+a+\mu) I(t)\right] d t \\
& +\sigma \frac{S(t) I(t)}{1+\alpha I(t)} d B(t), \\
& d R(t)=(\gamma I(t)-(\mu+\varepsilon) R(t)) d t .
\end{aligned}
$$

Throughout this paper, unless otherwise specified, let $(\Omega$, $\left.\left\{\mathbf{F}_{t}\right\}_{t \geq 0}, P\right)$ be a complete probability space with a filtration $\left\{\mathbf{F}_{t}\right\}_{t>0}$ satisfying the usual conditions (i.e., it is right continuous and $\left\{\mathbf{F}_{t}\right\}_{t \geq 0}$ contains all $P$-null sets) and let $B(t)$ be the Brownian motion defined on the probability space.

For simplicity, define

$$
\langle x(t)\rangle=\frac{1}{t} \int_{0}^{t} x(r) d r .
$$

\section{Existence and Uniqueness of the Nonnegative Solution}

In this section, we will show that there is a unique positive solution of system (4).
Theorem 1. For any initial value $(S(0), I(0), R(0))$ in $R_{+}^{3}$, there is a unique solution $(S(t), I(t), R(t))$ of system (4) on $t \geq 0$, and the solution will remain in $R_{+}^{3}$ with probability 1 .

Proof. Since the coefficients of system (4) are locally Lipschitz continuous, for any initial value $(S(0), I(0), R(0)) \in R_{+}^{3}$, there is a unique local solution on $\left[0, \tau_{e}\right)$, where $\tau_{e}$ is the explosion time. To show that this solution is global, we need to have $\tau_{e}=\infty$ a.s. To show that this solution is global, we need to have $\tau_{e}=\infty$ a.s. Let $m_{0} \geq 0$ be sufficiently large so that $(S(0), I(0), R(0))$ all lie in the interval $\left[1 / m_{0}, m_{0}\right]$. For each integer $m \geq m_{0}$, define the stopping time

$$
\begin{aligned}
\tau_{m} & =\inf \left\{t \in\left[0, \tau_{e}\right): \min \{S(t), I(t), R(t)\}\right. \\
& \left.\leq \frac{1}{m} \text { or } \max \{S(t), I(t), R(t)\} \geq 1\right\},
\end{aligned}
$$

where throughout this paper we set inf $\phi=\infty$ (as usual $\phi$ denotes the empty set). Clearly, $\tau_{m}$ is increasing as $m \rightarrow \infty$. Set $\tau_{\infty}=\lim _{t \rightarrow+\infty} \tau_{m}$, whence $\tau_{\infty} \leq \tau_{e}$ a.s. If we can show that $\tau_{\infty}=\infty$ a.s., then $\tau_{\infty}=\infty$ and $(S(0), I(0), R(0)) \in R_{+}^{3}$ a.s. for all $t \geq 0$. In other words, to complete the proof all we need to show is that $\tau_{\infty}=\infty$ a.s. If this statement is false, then there is a pair of constants $T>0$ and $\epsilon \in(0,1)$ such that $P\left\{\tau_{\infty} \leq T\right\}>\epsilon$.

Hence there is an integer $m_{1} \geq m_{0}$ such that

$$
P\left\{\tau_{\infty} \leq T\right\}>\epsilon, \quad \forall m \geq m_{1} .
$$

Besides, the total biomass $N(t)=S(t)+I(t)+R(t)$ of model (4) satisfies the following equation:

$$
d N(t)=[\Lambda-(\mu-b) N-a I] d t .
$$

It is easy to know that, for all $t<\tau_{\infty}$,

$$
N(t) \leq \max \left\{S_{0}, I_{0}, R_{0}, \frac{\Lambda}{\mu-b}\right\}:=K_{1} .
$$

Define a $C^{2}$-function $\mathrm{V}: \mathbb{R}_{+}^{3} \rightarrow \overline{\mathbb{R}}_{+}$by

$$
V(S, I, R)=-\log \frac{S}{K_{1}}-\log \frac{I}{K_{1}}-\log \frac{R}{K_{1}} .
$$

By using Itô's formula, we get

$$
d V=L V d t+\frac{\sigma(S-I)}{1+\alpha I},
$$

where

$$
\begin{aligned}
L V= & -\frac{\Lambda}{S}-b\left(1+\frac{R}{S}\right)-(1-q) b \frac{I}{S}+\frac{\beta I}{1+\alpha I}+\mu \\
& -\varepsilon \frac{R}{S}-\frac{\beta S}{1+\alpha I}+(\gamma+a+\mu-q b)-\frac{\gamma I}{R} \\
& +(\mu+\varepsilon)+\frac{\sigma^{2}\left(I^{2}+S^{2}\right)}{2(1+\alpha I)^{2}} \\
\leq & \frac{\beta I}{1+\alpha I}+(3 \mu+\gamma+a+\varepsilon-q b)+\frac{\sigma^{2}\left(I^{2}+S^{2}\right)}{2(1+\alpha I)^{2}} \\
\leq & \beta K_{1}+(3 \mu+\gamma+a+\varepsilon-q b)+K_{1}^{2} \sigma^{2}:=K_{2} .
\end{aligned}
$$


The remainder of the proof follows that in Li and Mao [21, Theorem 2.1].

Since there is a positive solution $(S(t), I(t), R(t)) \geq 0$ of system (4) for any given initial value $(S(0), I(0), R(0)) \in R_{+}^{3}$ and

$$
\begin{aligned}
\Gamma & =\left\{(S(t), I(t), R(t)) \in R_{+}^{3}: S(t)+I(t)+R(t)\right. \\
& \left.\leq \frac{\Lambda}{\mu-b}, t \geq 0 \text { a.s. }\right\}
\end{aligned}
$$

is an invariant set [22], then from now on, we can assume that $(S(0), I(0), R(0)) \in \Gamma$.

\section{Extinction}

In this section, we discuss the conditions for the extinction of the disease.

Theorem 2. Let $(S(t), I(t), R(t))$ be the solution of system (4) with initial value $(S(0), I(0), R(0)) \in \Gamma$. If $\widetilde{R_{0}}<1$ and $\sigma^{2} \leq$ $\beta(\mu-b) / \Lambda$ then

$$
\limsup _{t \rightarrow+\infty} \frac{\log I(t)}{t} \leq(\mu+a+\gamma-q b)\left(\widetilde{R_{0}}-1\right)<0
$$

Namely, $I(t)$ tends to zero exponentially a.s. where

$$
\widetilde{R_{0}}=R_{0}-\frac{\sigma^{2} \Lambda^{2}}{2(\mu-b)^{2}(\mu+a+\gamma-q b)}
$$

Proof. Notice that $S /(1+\alpha I) \in(0(\Lambda /(\mu-b))]$; that is, $\sigma^{2} \leq$ $\beta(\mu-b) / \Lambda$ the quadratic function

$$
f(z)=-\frac{\sigma^{2}}{2} z^{2}+\beta z-(\mu+a+\gamma-q b)
$$

gets its maximum value $f_{\max }$ on the interval $[0, \Lambda /(\mu-b)]$ at $z=\Lambda /(\mu-b)$, where

$$
\begin{aligned}
f_{\max } & =-\frac{\sigma^{2}}{2}\left(\frac{\Lambda}{\mu-b}\right)^{2}+\beta \frac{\Lambda}{\mu-b}-(\mu+a+\gamma-q b) \\
& =(\mu+\gamma+a-q b)\left(\widetilde{R_{0}}-1\right) .
\end{aligned}
$$

It follows from the monotonicity of the function $\beta S /(1+\alpha I)$ on $[0, \Lambda /(\mu-b)]$ that when $\sigma^{2} \leq \beta(\mu-b) / \Lambda$, we get

$$
\begin{aligned}
- & \frac{\sigma^{2}}{2}\left(\frac{S}{1+\alpha I}\right)^{2}+\beta \frac{S}{1+\alpha I}-(\mu+a+\gamma-q b) \\
& \leq(\mu+\gamma+a-q b)\left(\widetilde{R_{0}}-1\right) .
\end{aligned}
$$

Applying Itô's formula to system (4) leads to

$$
\begin{gathered}
d(\log I)=\left[-\frac{\sigma^{2}}{2}\left(\frac{S}{(1+\alpha I)}\right)^{2}+\beta \frac{S}{1+\alpha I}\right. \\
-(\mu+a+\gamma-q b)] d t+\frac{\sigma S}{1+\alpha I} d B(t) .
\end{gathered}
$$

Integrating this from 0 to $t$ and diving $t$ on both sides, we have

$$
\begin{aligned}
\frac{\log I(t)-\log I(0)}{t} \leq & (\mu+\gamma+a-q b)\left(\widetilde{R_{0}}-1\right) \\
& +\frac{M(t)}{t},
\end{aligned}
$$

where $M(t):=\int_{0}^{t}(\sigma S(r) /(1+\alpha I(r))) d B(r)$, which is local martingale and $M(0)=0$. Moreover,

$$
\limsup _{t \rightarrow+\infty} \frac{\langle M, M\rangle_{t}}{t} \leq \frac{\sigma^{2} \Lambda^{2}}{(\mu-b)^{2}}<\infty \text { a.s. }
$$

According to the large number theorem for martingales (see, e.g., [13]), we obtain

$$
\lim _{t \rightarrow+\infty} \frac{M(t)}{t}=0
$$

Then

$$
\limsup _{t \rightarrow+\infty} \frac{\log I(t)}{t} \leq(\mu+\gamma+a-q b)\left(\widetilde{R_{0}}-1\right)<0 \text { a.s. }
$$

The proof of Theorem 2 is completed.

Remark 3. We also notice that if $\beta / \sigma^{2} \in(0(\Lambda /(\mu-b))]$, that is, $\sigma^{2} \geq \beta(\mu-b) / \Lambda$, the quadratic function $f(z)$ obtains the maximum value $\beta^{2} / 2 \sigma^{2}-(\mu+\gamma+a-q b)$ at $z=\beta / \sigma^{2}$. The proof of Theorem 2 proceeded; we have

$$
\limsup _{t \rightarrow+\infty} \frac{\log I(t)}{t} \leq \frac{\beta^{2}}{2 \sigma^{2}}-(\mu+\gamma+a-q b)<0 \text { a.s. }
$$

if $\sigma^{2}>\beta^{2} / 2(\mu+a+\gamma-q b)$. It means that the disease will always die out if the noise is large enough such that $\sigma^{2}>\max \{\beta(\mu-$ b) $\left./ \Lambda, \beta^{2} / 2(\mu+a+\gamma-q b)\right\}$.

Remark 4. From Theorem 2, we can get that the disease will die out if $\widetilde{R_{0}}<1$ and the white noise is not large such that $\sigma^{2} \leq$ $\beta(\mu-b) / \Lambda$. Meanwhile if white noise is large enough such that $\sigma^{2}>\max \left\{\beta(\mu-b) / \Lambda, \beta^{2} / 2(\mu+a+\gamma-q b)\right\}$ is satisfied, then the disease will also die out. Moreover, we notice $\widetilde{R_{0}}$ is smaller than the threshold of the corresponding deterministic model. The following examples illustrate this result more explicitly.

From Theorem 2, it is obvious that under some conditions

$$
\limsup _{t \rightarrow+\infty} \frac{\log I(t)}{t}<0 \text { a.s. }
$$

which implies

$$
\lim _{t \rightarrow \infty} I(t)=0 \text { a.s. }
$$


Next, according to system (4), we get

$$
\begin{aligned}
& d(S(t)+I(t)+R(t)) \\
& \quad=[\Lambda-(\mu-b)(S(t)+I(t)+R(t))-a I] d t \\
& \quad \geq[\Lambda-(\mu-b)(S(t)+I(t)+R(t))] d t
\end{aligned}
$$

which implies that

$$
\liminf _{t \rightarrow+\infty}(S(t)+I(t)+R(t)) \geq \frac{\Lambda}{\mu-b} \text { a.s. }
$$

Together with (13), we have

$$
\lim _{t \rightarrow+\infty}(S(t)+R(t))=\frac{\Lambda}{\mu-b} \text { a.s. }
$$

According to (26) and the last equation of system (4), we obtain

$$
\lim _{t \rightarrow \infty} R(t)=0 \text { a.s. }
$$

Therefore, by (29), we have

$$
\lim _{t \rightarrow+\infty} S(t)=\frac{\Lambda}{\mu-b} \text { a.s. }
$$

We have the following theorem by combing these arguments.

Theorem 5. Let $(S(t), I(t), R(t))$ be the solution of system (4) with initial value $(S(0), I(0), R(0)) \in \Gamma$. If

(i) $\widetilde{R_{0}}<1$ and $\sigma^{2} \leq \beta(\mu-b) / \Lambda$ or

(ii) $\sigma^{2}>\max \left\{\beta(\mu-b) / \Lambda, \beta^{2} / 2(\mu+a+\gamma-q b)\right\}$ then

$$
\begin{aligned}
& \lim _{t \rightarrow+\infty} S(t)=\frac{\Lambda}{\mu-b}, \\
& \lim _{t \rightarrow+\infty} I(t)=0, \\
& \lim _{t \rightarrow+\infty} R(t)=0 \text { a.s. }
\end{aligned}
$$

Example 6. We assume that the unit of time is one day and the population sizes are measured in units of 1 million. The parameters in system (4) are chosen as follows:

$$
\begin{aligned}
& \Lambda=0.5, \\
& \beta=0.8, \\
& \mu=0.3, \\
& b=0.05, \\
& \alpha=0.2, \\
& q=0.2, \\
& \gamma=0.39, \\
& a=0.21, \\
& \varepsilon=0.4, \\
& \sigma=0.5 .
\end{aligned}
$$

Note that

$$
\begin{aligned}
& \widetilde{R_{0}}=0.6742<1 \\
& \sigma^{2}=0.25 \leq \frac{\beta(\mu-b)}{\Lambda}=0.4
\end{aligned}
$$

and then by Theorem 5(i), the solution $(S(t), I(t), R(t))$ of system (4) obeys

$$
\begin{aligned}
\limsup _{t \rightarrow+\infty} \frac{\log I(t)}{t} & \leq(\mu+\gamma+a-q b)\left(\widetilde{R_{0}}-1\right)=-0.41 \\
& <0 \text { a.s. } \\
\lim _{t \rightarrow+\infty} S(t) & =\frac{\Lambda}{\mu-b}=2 \\
\lim _{t \rightarrow+\infty} R(t) & =0 \text { a.s. }
\end{aligned}
$$

with any initial value $(S(0), I(0), R(0))=(0.6,0.4,0.5) \in \Gamma$. Then the disease tends to zero exponentially with probability one.

On the other hand, for the responding deterministic SIRS model, $R_{0}=1.7978>1$; the disease will prevail. Using the method mentioned in [23], we provide the simulations shown in Figure 1 to support our results.

Example 7. We choose the same values of the parameters of (33) except for the value of $\sigma$. Because $\sigma^{2}>\beta(\mu-b) / \Lambda=$ $0.4, \sigma^{2}>\beta^{2} / 2(\mu+\gamma+a-q b)=0.3595$, we let $\sigma=$ 0.7 ; then by Theorem 5(ii), the solution of system (4) obeys $\lim \sup _{t \rightarrow+\infty}(\log I(t) / t) \leq-(\mu+\gamma+a-q b)+\beta^{2} / 2 \sigma^{2}=$ $-0.2369<0$ a.s., in which case the disease will also die out. Note that $R_{0}=1.2584>1$, so that the disease for the corresponding system will prevail too (see Figure 2).

\section{Persistence}

Definition 8. System (4) is said to be persistent in the mean if

$$
\lim _{t \rightarrow+\infty} \frac{1}{t} \int_{0}^{t} I(r) d r>0 \text { a.s. }
$$

Theorem 9. If $\widetilde{R_{0}}>1$, then for any initial value $(S(0)$, $I(0), R(0)) \in \Gamma$, the solution $(S(t), I(t), R(t))$ of system (4) has the following property: 

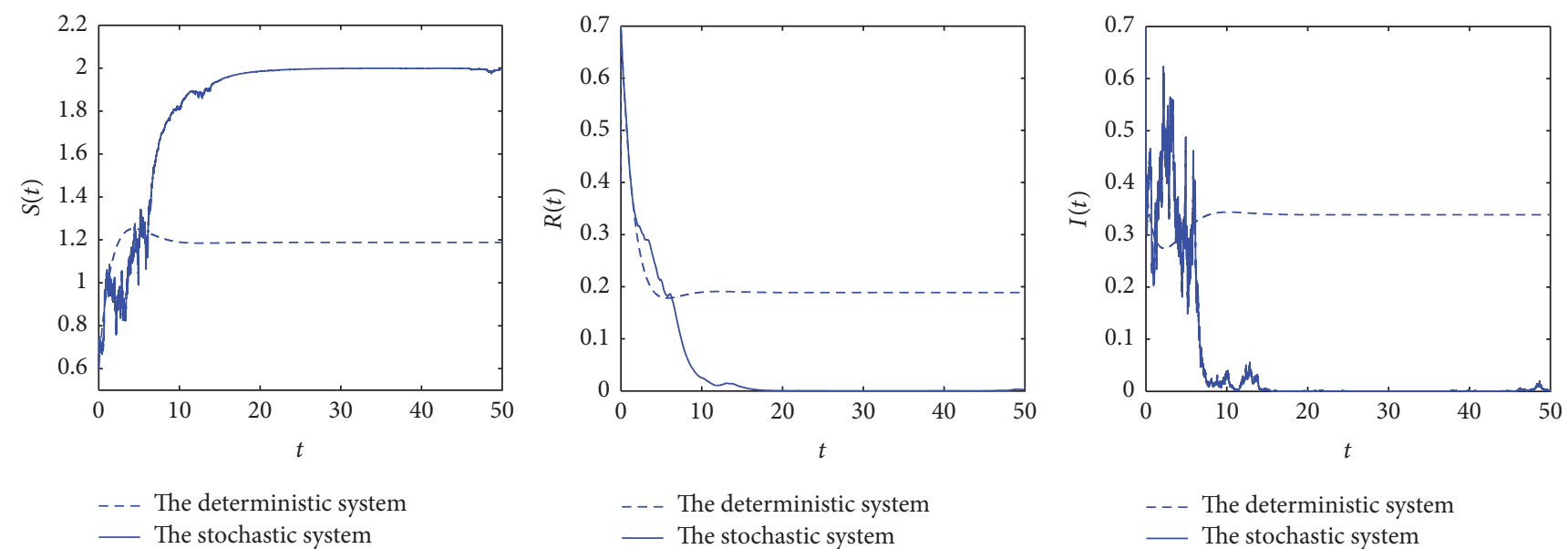

FIGURE 1: Simulations of the path $(S(t), I(t), R(t))$ for the corresponding deterministic system (2) and stochastic system (4) with initial value $(S(0), I(0), R(0))=(0.6,0.4,0.5), \sigma=0.5$.
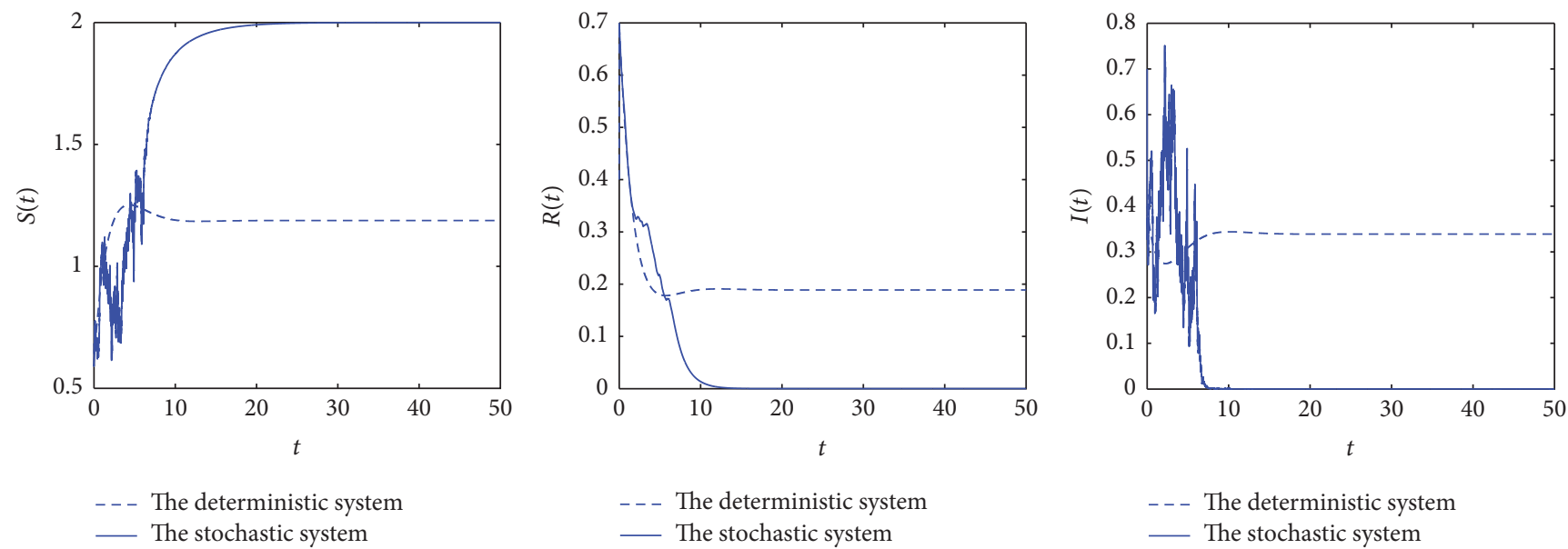

FIGURE 2: Simulations of the path $S(t), I(t), R(t)$ for the corresponding deterministic system (2) and stochastic system (4) with initial value $(S(0), I(0), R(0))=(0.6,0.4,0.5), \sigma=0.7$.

$$
\liminf _{t \rightarrow+\infty}\langle I(t)\rangle \geq \frac{(\mu+a+\gamma-q b)\left(\widetilde{R_{0}}-1\right)}{\beta(((\mu+\varepsilon)(\mu+a+\gamma-q b)-\gamma(b+\varepsilon)) /(\mu+\varepsilon)(\mu-b))+(\mu+a+\gamma-q b)}:=I_{*} \text { a.s. }
$$

Proof. An integration of system (4) yields

$$
\begin{aligned}
& -\frac{\sigma}{t} \int_{0}^{t} \frac{S(r) I(r)}{1+\alpha I(r)} d B(r), \\
\frac{I(t)-I(0)}{t}= & \beta\left\langle\frac{S(t) I(t)}{1+\alpha I(t)}\right\rangle \\
& -(\mu+a+\gamma-q b)\langle I(t)\rangle \\
& +\frac{\sigma}{t} \int_{0}^{t} \frac{S(r) I(r)}{1+\alpha I(r)} d B(r), \\
\frac{R(t)-R(0)}{t}= & \gamma\langle I(t)\rangle-(\mu+\varepsilon)\langle R(t)\rangle .
\end{aligned}
$$$$
\frac{S(t)-S(0)}{t}=\Lambda+b\langle S(t)\rangle+b\langle R(t)\rangle
$$$$
+(1-q) b\langle I(t)\rangle
$$$$
-\beta\left\langle\frac{S(t) I(t)}{1+\alpha I(t)}\right\rangle-\mu\langle S(t)\rangle
$$$$
+\varepsilon\langle R(t)\rangle
$$ 
According to (39), we have

$$
\begin{aligned}
\frac{1}{b+\varepsilon} & \left(\frac{S(t)-S(0)}{t}+\frac{I(t)-I(0)}{t}\right) \\
& +\frac{1}{\mu+\epsilon} \frac{R(t)-R(0)}{t} \\
= & \frac{\Lambda}{b+\varepsilon}-\frac{\mu-b}{b+\varepsilon}\langle S(t)\rangle \\
& +\left(\frac{\gamma}{\mu+\varepsilon}-\frac{\mu+\gamma+a-b}{b+\varepsilon}\right)\langle I(t)\rangle .
\end{aligned}
$$

After simple computing

$\langle S(t)\rangle$

$$
\begin{aligned}
= & \frac{\Lambda}{\mu-b} \\
& -\frac{(\mu+\varepsilon)(\mu+a+\gamma-q b)-\gamma(b+\varepsilon)}{(\mu+\varepsilon)(\mu-b)}\langle I(t)\rangle \\
& +\varphi(t),
\end{aligned}
$$

where $\varphi(t)=-((b+\varepsilon) /(\mu-b))[(1 /(b+\varepsilon))((S(t)-S(0)) / t+(I(t)-$ $I(0)) / t)+(1 /(\mu+\epsilon))((R(t)-R(0)) / t)]$ and $\lim _{t \rightarrow+\infty} \varphi(t)=$ 0 a.s.

Let $X=((\mu+\varepsilon)(\mu+a+\gamma-q b)-\gamma(b+\varepsilon)) /(\mu+\varepsilon)(\mu-b)$.

By Itô's formula, we obtain

$$
\begin{aligned}
& d(\log I+\alpha I)=[\beta S-(\mu+a+\gamma-q b) \\
& \left.-\frac{\sigma^{2} S^{2}}{2(1+\alpha I)^{2}}-(\mu+a+\gamma-q b) I\right] d t \\
& +\sigma S d B(t) \geq\left[\beta S-(\mu+a+\gamma-q b)-\frac{\sigma^{2} \Lambda^{2}}{2(\mu-b)^{2}}\right. \\
& -(\mu+a+\gamma-q b) I] d t+\sigma S d B(t)
\end{aligned}
$$

and then

$$
\begin{gathered}
\frac{\log I(t)-\log I(0)}{t} \geq[\beta\langle S(t)\rangle-(\mu+a+\gamma-q b) \\
\left.-\frac{\sigma^{2} \Lambda^{2}}{2(\mu-b)^{2}}-(\mu+a+\gamma-q b)\langle I(t)\rangle\right] d t+\frac{\sigma}{t} \\
\cdot \int_{0}^{t} S(r) d B(r)-\alpha \frac{I(t)-I(0)}{t} \geq \beta \frac{\Lambda}{\mu-b}
\end{gathered}
$$

$$
\begin{aligned}
& +\beta \varphi(t)-(\mu+a+\gamma-q b)-[\beta X \\
& +(\mu+a+\gamma-q b)]\langle I(t)\rangle-\frac{\sigma^{2} \Lambda^{2}}{2(\mu-b)^{2}} d t+\frac{\sigma}{t} \\
& \cdot \int_{0}^{t} S(r) d B(r)-\alpha \frac{I(t)-I(0)}{t}=(\mu+a \\
& +\gamma-q b)\left(\widetilde{R_{0}}-1\right)+\beta \varphi(t)-[\beta X \\
& +(\mu+a+\gamma-q b)]\langle I(t)\rangle d t+\frac{\sigma}{t} \int_{0}^{t} S(r) d B(r) \\
& -\alpha \frac{I(t)-I(0)}{t} .
\end{aligned}
$$

Then the inequality can be rewritten as

$$
\begin{aligned}
& \langle I(t)\rangle \geq \frac{1}{\beta X+(\mu+a+\gamma-q b)}[(\mu+a+\gamma-q b) \\
& \cdot\left(\widetilde{R_{0}}-1\right)+\beta \varphi(t)-\frac{\log I(t)-\log I(0)}{t}-\alpha \\
& \left.\cdot \frac{I(t)-I(0)}{t}+\frac{M(t)}{t}\right] .
\end{aligned}
$$

By (29), then taking the limit inferior of both sides (44) leads to

$$
\liminf _{t \rightarrow+\infty}\langle I(t)\rangle \geq \frac{(\mu+a+\gamma-q b)\left(\widetilde{R_{0}}-1\right)}{\beta X+(\mu+a+\gamma-q b)}:=I_{*} .
$$

This completes the proof of Theorem 9.

Example 10. Choose the parameters in system (4) as follows:

$$
\begin{aligned}
& \Lambda=0.5, \\
& \beta=0.8, \\
& \mu=0.3, \\
& b=0.05, \\
& \alpha=0.2, \\
& q=0.2, \\
& \gamma=0.39, \\
& a=0.21, \\
& \varepsilon=0.4, \\
& \sigma=0.2 .
\end{aligned}
$$

Note that

$$
\begin{aligned}
& \widetilde{R_{0}}=1.7078>1, \\
& \sigma^{2}=0.04 \leq \frac{\beta(\mu-b)}{\Lambda}=0.4 .
\end{aligned}
$$



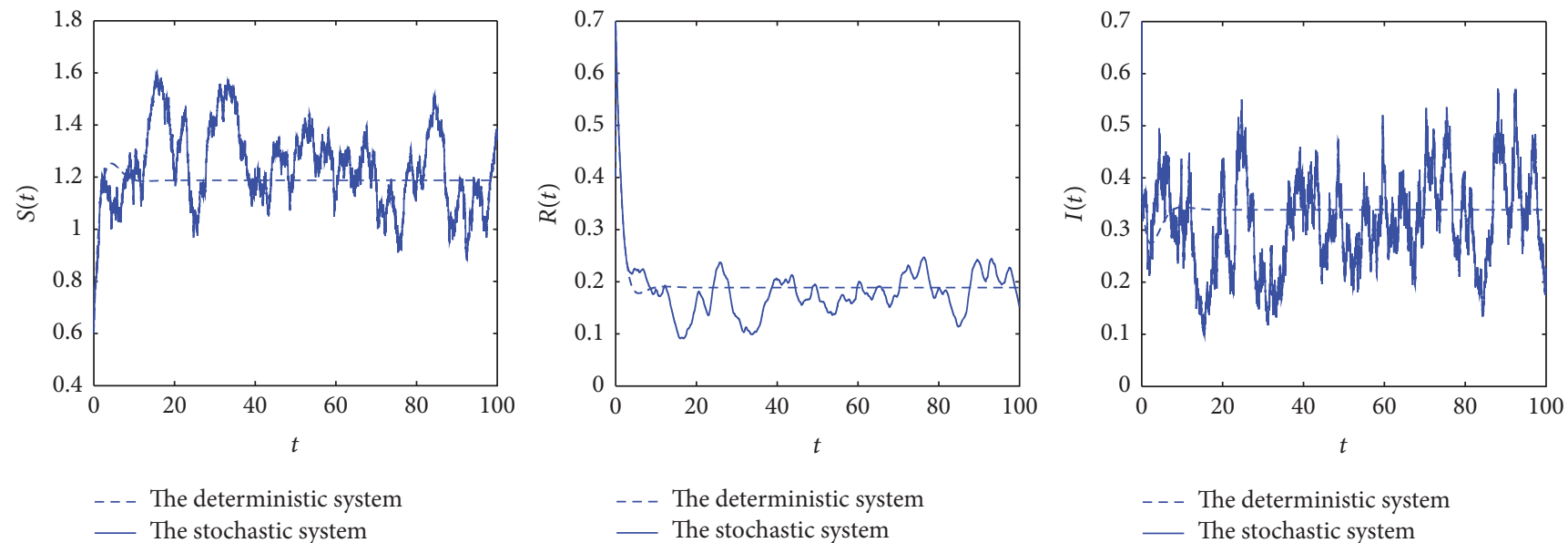

FIGURE 3: Simulations of the path $S(t), I(t), R(t)$ for the corresponding deterministic system (2) and stochastic system (4) with initial value $(S(0), I(0), R(0))=(0.6,0.4,0.5), \sigma=0.2$.
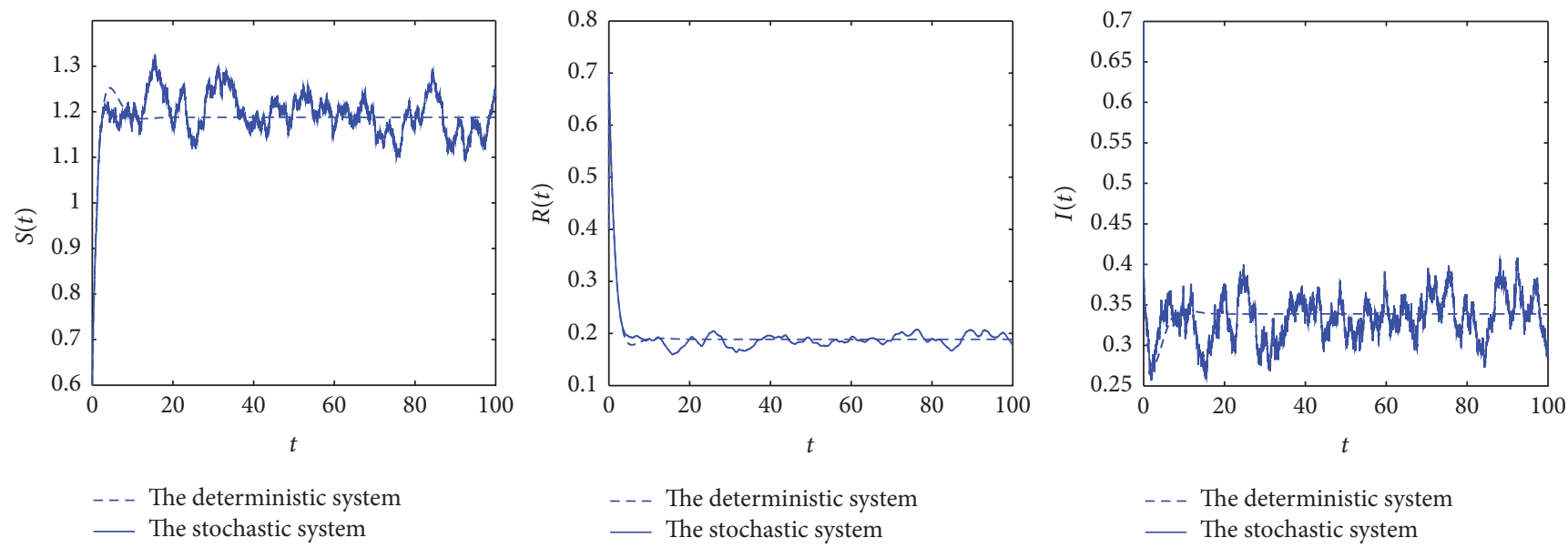

FIGURE 4: Simulations of the path $S(t), I(t), R(t)$ for the corresponding deterministic system (2) and stochastic system (4) with initial value $(S(0), I(0), R(0))=(0.6,0.4,0.5), \sigma=0.06$.

and then by Theorems 2, with any initial value $(S(0)$, $I(0), R(0))=(0.6,0.4,0.5) \in \Gamma$, we get that the solution of system (4) obeys

$$
\liminf _{t \rightarrow+\infty}\langle I(t)\rangle \leq 0.2146 \text { a.s. }
$$

Using the method mentioned in [23], we give the simulations shown in Figure 3, and we find the disease is persistent.

Example 11. We consider the same values of the parameters of (46) except $\sigma$. Change $\sigma$ to 0.06 to further illustrate the effect of the noise intensity $\sigma$ on this SIRS model. Notice that $\widetilde{R_{0}}=1.7816>1$ and $\sigma^{2}=(0.06)^{2} \leq \beta(\mu-b) / \Lambda=0.4$ Using the method mentioned in [14], we give the simulations shown in Figure 4 to support our results. And we find the fluctuation of the solution of system (4) is becoming weaker compared with the picture in Figure 3.

\section{Conclusion}

In this paper, we study a stochastic SIRS model with vertical transmission and saturated incidence. We find that the extinction or persistence of the disease is mainly determined by the value of $\widetilde{R_{0}}$, and it is obvious that $\widetilde{R_{0}}<R_{0}$ when $\sigma \neq 0$. We find when the noise is so small (in fact $\sigma^{2}<$ $\beta(\mu-b) / \Lambda)$, the extinction and persistence are completely determined by $\widetilde{R_{0}}$ : if $\widetilde{R_{0}}<1$, the disease dies out; if $\widetilde{R_{0}}>1$, the disease prevails. So the noise is adverse to the survival of the disease. We also find that if the noise is so large that $\sigma^{2}>\max \left\{\beta(\mu-b) / \Lambda, \beta^{2} / 2(\mu+a+\gamma-q b)\right\}$, the disease prevails too.

To understand how the noise effects the survival of the disease better, the conditions in Theorem 2 can be written as

$$
\begin{aligned}
\sigma & >\frac{1}{\Lambda} \sqrt{2(\mu-b)[\beta \Lambda-(\mu-b)(\mu+a+\gamma-q b)]} \\
& :=\widetilde{\sigma} \\
\sigma & \leq \frac{1}{\Lambda} \sqrt{\beta \Lambda(\mu-b)},
\end{aligned}
$$

and rewrite the condition $\widetilde{R_{0}}>1$ in Theorem 5 as follows: $\sigma<\tilde{\sigma}$. 
We obtain the main results as follows:

(a) If $R_{0}<1$, the disease will always die out without considering the existence of the noise according to (15).

(b) If $R_{0}>1$, the disease will persist for the deterministic model (2) while for the stochastic model (4), the disease will persist when $\sigma<\widetilde{\sigma}$ and die out when $\tilde{\sigma}<\sigma \leq(1 / \Lambda) \sqrt{\beta \Lambda(\mu-b)}$. The existence of noise determines the extinction of the disease.

(c) If $\widetilde{\sigma}>\max \{(1 / \Lambda) \sqrt{\beta \Lambda(\mu-b)},(\beta / 2(\mu+a+\gamma-$ $q b)) \sqrt{2(\mu+a+\gamma-q b)}\}$, the disease will always die out.

From (b) we get that when $R_{0}>1, \widetilde{\sigma}=\sigma$ is the threshold which determines the extinction and persistence of the disease for stochastic system (4). Meanwhile, we can obtain from cases (b) and (c) that if

$$
\begin{gathered}
\max \left\{\frac{1}{\Lambda} \sqrt{\beta \Lambda(\mu-b)}, \frac{\beta}{2(\mu+a+\gamma-q b)}\right. \\
\cdot \sqrt{2(\mu+a+\gamma-q b)}\}=\frac{1}{\Lambda} \sqrt{\beta \Lambda(\mu-b)},
\end{gathered}
$$

then disease will die out when $\sigma>\tilde{\sigma}$; meanwhile if

$$
\begin{aligned}
& \max \left\{\frac{1}{\Lambda} \sqrt{\beta \Lambda(\mu-b)}, \frac{\beta}{2(\mu+a+\gamma-q b)}\right. \\
& \cdot \sqrt{2(\mu+a+\gamma-q b)}\}=\frac{\beta}{2(\mu+a+\gamma-q b)} \\
& \cdot \sqrt{2(\mu+a+\gamma-q b)},
\end{aligned}
$$

the disease will die out only for $\sigma \in(\widetilde{\sigma},(1 / \Lambda) \sqrt{\beta \Lambda(\mu-b)}) \cup$ $((\beta / 2(\mu+a+\gamma-q b)) \sqrt{2(\mu+a+\gamma-q b)}, \infty)$.

\section{Competing Interests}

The authors declare that they have no competing interests.

\section{Acknowledgments}

The research was supported by the Project for Science and Technology in Shaoguan (2014CX/K231) and the HighLevel Talents Project of Guangdong Province Colleges and Universities (2013-178).

\section{References}

[1] H. W. Hethcote, "Qualitative analyses of communicable disease models," Mathematical Biosciences, vol. 28, no. 3-4, pp. 225-356, 1976.

[2] S. M. Blower, A. R. McLean, T. C. Porco et al., "The intrinsic transmission dynamics of tuberculosis epidemics," Nature Medicine, vol. 1, no. 8, pp. 815-821, 1995.
[3] T. Lietman and S. M. Blower, "Potential impact of tuberculosis vaccines as epidemic control agents," Clinical Infectious Diseases, vol. 30, no. 3, pp. S316-S322, 2000.

[4] E. Ziv, C. L. Daley, and S. Blower, "Potential public health impact of new tuberculosis vaccines," Emerging Infectious Diseases, vol. 10, no. 9, pp. 1529-1535, 2004.

[5] C. Vargas-De-Len, "On the golbal stability of SIS, SIR and SIRS epidemic models incidence rate," Journal of Differential Equations, vol. 188, pp. 135-163, 2003.

[6] M. Fan, M. Y. Li, and K. Wang, "Global stability of an SEIS epidemic model with recruitment and a varying total population size," Mathematical Biosciences, vol. 170, no. 2, pp. 199-208, 2001.

[7] L.-M. Cai, X.-Z. Li, and M. Ghosh, "Global stability of a stagestructured epidemic model with a nonlinear incidence," Applied Mathematics and Computation, vol. 214, no. 1, pp. 73-82, 2009.

[8] D. Z. Gao and S. G. Ruan, "An SIS patch model with variable transmission coefficients," Mathematical Biosciences, vol. 232, no. 2, pp. 110-115, 2011.

[9] Y. Muroya, Y. Enatsu, and Y. Nakata, "Global stability of a delayed SIRS epidemic model with a non-monotonic incidence rate," Journal of Mathematical Analysis and Applications, vol. 377, no. 1, pp. 1-14, 2011.

[10] M. E. Alexander and S. M. Moghadas, "Bifurcation analysis of SIRS epidemic model with generalized incidence," SIAM Journal on Applied Mathematics, vol. 65, no. 5, pp. 1794-1816, 2005.

[11] X. Song, Y. Jiang, and H. Wei, "Analysis of a saturation incidence SVEIRS epidemic model with pulse and two time delays," Applied Mathematics and Computation, vol. 214, no. 2, pp. 381390, 2009.

[12] M. Bandyopadhyay and J. Chattopadhyay, "Ratio-dependent predator-prey model: effect of environmental fluctuation and stability," Nonlinearity, vol. 18, no. 2, pp. 913-936, 2005.

[13] L. J. Allen and N. Kirupaharan, "Asymptotic dynamics of deterministic and stochastic epidemic models with multiple pathogens," International Journal of Numerical Analysis and Modeling, vol. 2, no. 3, pp. 329-344, 2005.

[14] Y. Zhao and D. Jiang, "The threshold of a stochastic SIRS epidemic model with saturated incidence," Applied Mathematics Letters, vol. 34, pp. 90-93, 2014.

[15] L. J. S. Allen and P. van den Driessche, "Stochastic epidemic models with a backward bifurcation," Mathematical Biosciences and Engineering, vol. 3, no. 3, pp. 445-458, 2006.

[16] Y. Zhao, D. Jiang, X. Mao, and A. Gray, "The threshold of a stochastic SIRS epidemic model in a population with varying size," Discrete and Continuous Dynamical Systems. Series B, vol. 20, no. 4, pp. 1277-1295, 2015.

[17] Y. Zhao, D. Jiang, and D. O'Regan, “The extinction and persistence of the stochastic SIS epidemic model with vaccination," Physica A: Statistical Mechanics and Its Applications, vol. 392, no. 20, pp. 4916-4927, 2013.

[18] E. Tornatore, S. M. Buccellato, and P. Vetro, "Stability of a stochastic SIR system," Physica A: Statistical Mechanics and Its Applications, vol. 354, no. 1-4, pp. 111-126, 2005.

[19] G. Chen and T. Li, "Stability of stochastic delayed SIR model," Stochastics and Dynamics, vol. 9, no. 2, pp. 231-252, 2009.

[20] A. Gray, D. Greenhalgh, X. Mao, and J. Pan, “The SIS epidemic model with Markovian switching," Journal of Mathematical Analysis and Applications, vol. 394, no. 2, pp. 496-516, 2012. 
[21] X. Li and X. Mao, "Population dynamical behavior of nonautonomous Lotka-Volterra competitive system with random perturbation," Discrete and Continuous Dynamical Systems Series A, vol. 24, no. 2, pp. 523-545, 2009.

[22] C. Ji, D. Jiang, and N. Shi, "The behavior of an SIR epidemic model with stochastic perturbation," Stochastic Analysis and Applications, vol. 30, no. 5, pp. 755-773, 2012.

[23] D. J. Higham, "An algorithmic introduction to numerical simulation of stochastic differential equations," SIAM Review, vol. 43, no. 3, pp. 525-546, 2001. 


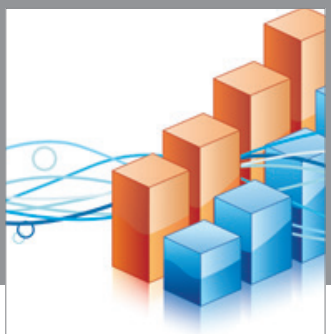

Advances in

Operations Research

vatem alat4

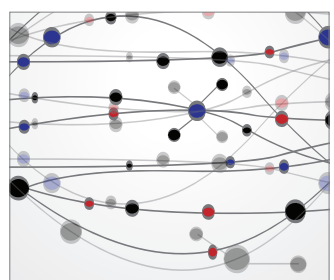

\section{The Scientific} World Journal
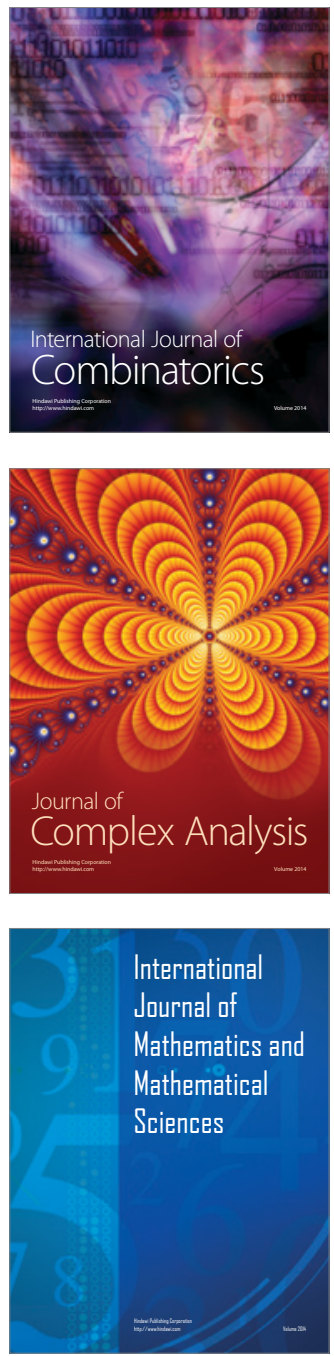
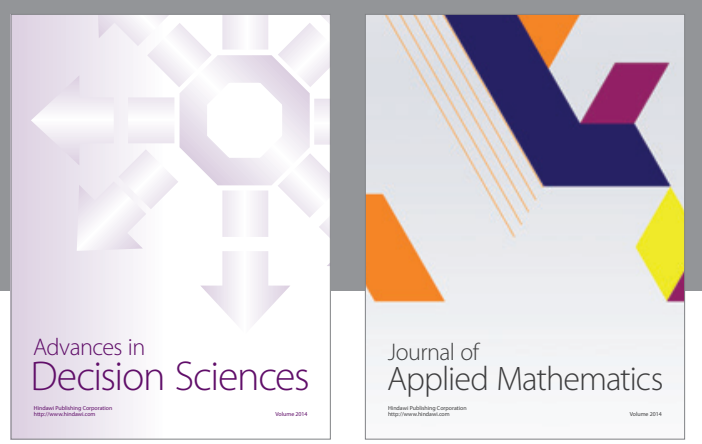

Algebra

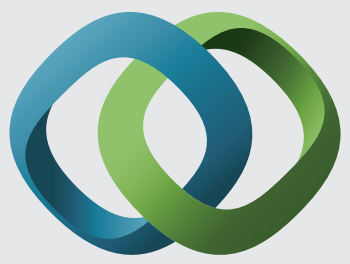

\section{Hindawi}

Submit your manuscripts at

https://www.hindawi.com
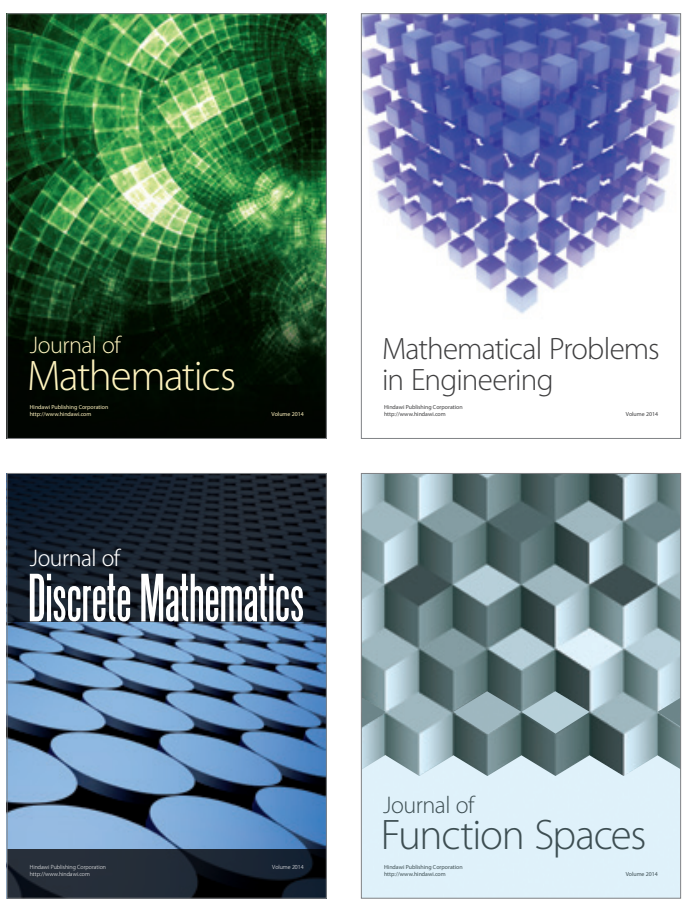

Mathematical Problems in Engineering
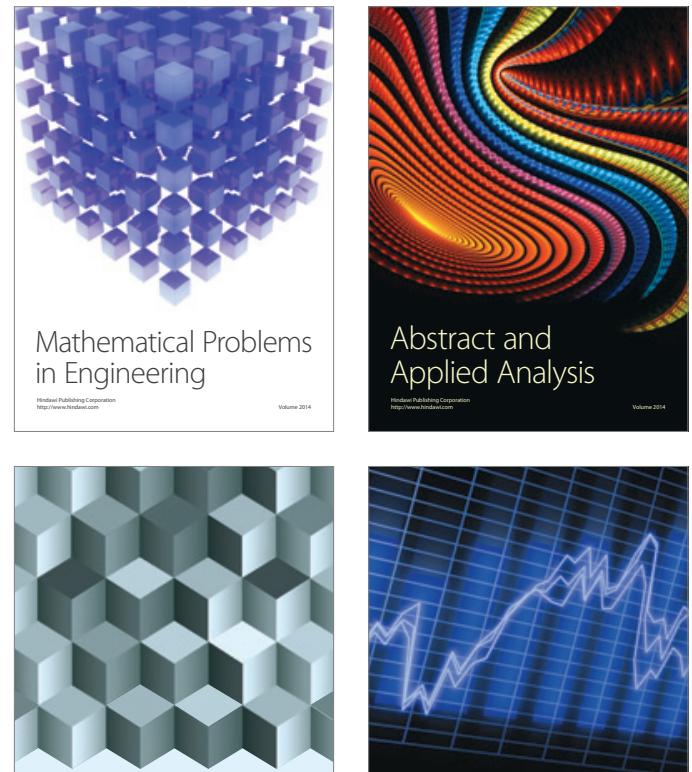

Journal of

Function Spaces

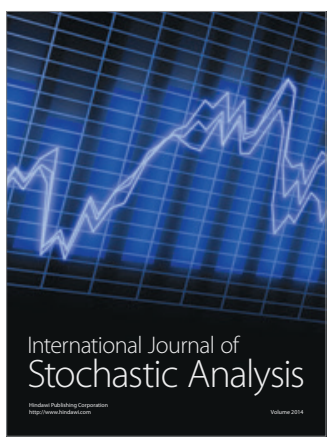

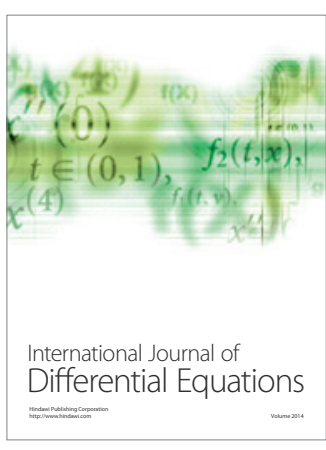
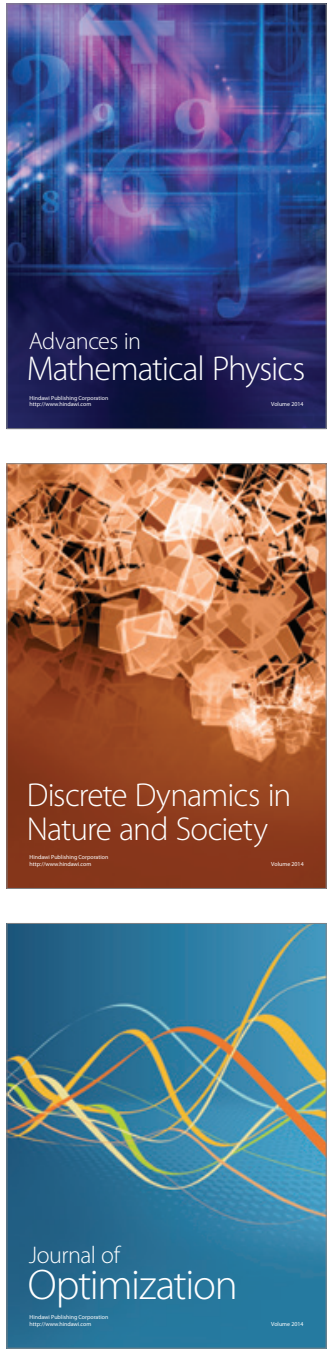